\title{
E2F7 regulates transcription and maturation of multiple microRNAs to restrain cell proliferation
}

\author{
Jone Mitxelena' ${ }^{1}$, Aintzane Apraiz ${ }^{2}$, Jon Vallejo-Rodríguez ${ }^{1}$, Marcos Malumbres $^{3}$ and Ana \\ M. Zubiaga ${ }^{1, *}$ \\ ${ }^{1}$ Department of Genetics, Physical Anthropology and Animal Physiology, University of the Basque Country \\ UPV/EHU, 48080 Bilbao, Spain, ${ }^{2}$ Department of Cell Biology and Histology, University of the Basque Country \\ UPV/EHU, 48080 Bilbao, Spain and ${ }^{3}$ Cell Division and Cancer Group, Spanish National Cancer Research Centre \\ (CNIO), 28029 Madrid, Spain
}

Received October 4, 2015; Revised February 29, 2016; Accepted March 1, 2016

\begin{abstract}
E2F transcription factors (E2F1-8) are known to coordinately regulate the expression of a plethora of target genes, including those coding for microRNAs (miRNAs), to control cell cycle progression. Recent work has described the atypical E2F factor E2F7 as a transcriptional repressor of cell cycle-related protein-coding genes. However, the contribution of E2F7 to miRNA gene expression during the cell cycle has not been defined. We have performed a genome-wide RNA sequencing analysis to identify E2F7-regulated miRNAs and show that E2F7 plays as a major role in the negative regulation of a set of miRNAs that promote cellular proliferation. We provide mechanistic evidence for an interplay between E2F7 and the canonical E2F factors E2F1-3 in the regulation of multiple miRNAs. We show that miR$25,-26 a,-27 b,-92 a$ and -7 expression is controlled at the transcriptional level by the antagonistic activity of E2F7 and E2F1-3. By contrast, let-7 miRNA expression is controlled indirectly through a novel E2F/C-MYC/LIN28B axis, whereby E2F7 and E2F1-3 modulate C-MYC and LIN28B levels to impact let-7 miRNA processing and maturation. Taken together, our data uncover a new regulatory network involving transcriptional and post-transcriptional mechanisms controlled by E2F7 to restrain cell cycle progression through repression of proliferation-promoting miRNAs.
\end{abstract}

\section{INTRODUCTION}

Since the initial identification of E2F as the cellular factor required for activation of the E2 adenoviral promoter, the E2F family of transcription factors has expanded through the addition of new members in mammals and through the discovery of homologs in other eukaryotes. Eight mammalian E2F family members (E2F1-8) have been identified, which orchestrate a complex gene regulatory network to ensure proper cell cycle progression, cellular differentiation and development $(1,2)$. However, it is still unclear what the precise roles of each individual E2F member are, and how the activity of the whole E2F family is coordinated to achieve an integrated regulation of gene expression.

Canonical E2F proteins (E2F1-6) bear one DNAbinding domain (DBD) immediately followed by a dimerization domain, which mediates interaction with the dimerization partner protein (DP). This dimerization enables E2Fs to bind DNA with high affinity, and to function as transcriptional regulators (3). According to the prevailing model, transcriptional regulation by canonical E2Fs is controlled through association with the retinoblastoma (RB) family of tumor suppressor proteins (pRB, p107 and p130) in the case of E2F1-5, or with polycomb group (PcG) proteins, in the case of E2F6 (4). These associations facilitate recruitment of histone deacetylases and methyltransferases to target promoters and subsequent transcriptional repression. Disruption of repressor complexes unleashes E2F activity, thereby triggering target gene transcription (3).

By contrast to canonical E2Fs, the atypical members E2F7 and E2F8, display two tandem DBDs and lack sequences that mediate RB and DP binding (5). The mechanisms by which atypical E2Fs regulate gene expression as well as their biological roles are still unclear. Gain-offunction experiments have revealed that $\mathrm{E} 2 \mathrm{~F} 7$ and $\mathrm{E} 2 \mathrm{~F} 8$ are recruited to promoters of several E2F target genes involved in DNA replication and DNA repair, and repress E2F sitedependent transcription in a RB-independent manner (611). Furthermore, overexpression of either E2F7 or E2F8 disrupts cell cycle progression, suggesting that they might promote negative cell cycle control through transcriptional repression of cell cycle genes (6-11). However, knockout

\footnotetext{
*To whom correspondence should be addressed. Tel: +34 94601 2603; Fax: +34 94601 3143; Email: ana.zubiaga@ehu.es

Present address: Jone Mitxelena, Department of Molecular Mechanisms of Disease, University of Zurich, Switzerland.

(C) The Author(s) 2016. Published by Oxford University Press on behalf of Nucleic Acids Research.

This is an Open Access article distributed under the terms of the Creative Commons Attribution License (http://creativecommons.org/licenses/by-nc/4.0/), which permits non-commercial re-use, distribution, and reproduction in any medium, provided the original work is properly cited. For commercial re-use, please contact journals.permissions@oup.com
} 
(KO) of E2F7 or E2F8 in mice has no significant effect on cell cycle progression, and a concomitant inactivation of E2F7 and E2F8 is needed to impact on cell cycle progression in vivo (12). This is probably due to compensatory mechanisms between both E2Fs, a common outcome in constitutive $\mathrm{KO}$ mouse models. Thus, the specific contribution of E2F7 and E2F8 to cell cycle control remains to be elucidated.

Significant progress in the understanding of E2Fmediated regulation of gene expression has been achieved by the finding that many microRNA-coding genes are bona fide E2F target genes (13-20). In line with the complex nature of the E2F pathway, many reports have uncovered an essential role for E2F-regulated microRNAs in modulating distinct cellular processes, most notably pathways involved in neoplastic transformation $(21,22)$. Some of these E2F-regulated miRNAs, including miR-17-92, miR-106b25, mir-15b-16-2 and miR-15a-16-1, appear to function as tumor suppressors that modulate and restrict progression through the cell cycle by limiting the expression of E2Fs themselves as well as other pathway components, thereby creating negative feedback loops $(14,16,18)$. By contrast, there is also evidence for an oncogenic potential for some E2F-dependent miRNAs. For instance, miR17-92 and miR-106b-25 clusters have been found to suppress the expression of anti-proliferative and pro-apoptotic genes, such as p21 $1^{\mathrm{CIP} 1}, \mathrm{pRB}, \mathrm{p} 130, \mathrm{p} 57^{\mathrm{KIP} 2}$, PTEN and BIM $(13,17,23-25)$. Given that each miRNA can regulate the expression of numerous genes, the list of genes regulated by miRNAs under E2F control is likely to include other, yet to be identified, targets.

The contribution of atypical E2F factors to miRNA expression regulation, and the effect that target miRNAs have on the biological roles mediated by E2F7 and E2F8, are still unknown. In this work, we have investigated the role of E2F7 in the regulation of miRNA-coding gene expression. We show that E2F7 is required for the timely repression of a set of miRNAs that function to promote cell proliferation. Importantly, our data uncover both transcriptional and post-transcriptional mechanisms for E2F7-mediated regulation of these miRNAs, and provide new insights to the understanding of E2F-regulated gene network.

\section{MATERIALS AND METHODS}

\section{Cell culture conditions and flow cytometry}

Human U2OS osteosarcoma cell line and human embryonic kidney (HEK) 293T cells were maintained in Dulbecco's modified Eagle's medium supplemented with 10\% fetal bovine serum (FBS). For cell synchronization in G1/S, exponentially growing cells were incubated with $4 \mathrm{mM}$ hydroxyurea (HU) for $24 \mathrm{~h}$ and subsequently washed and cultured in complete medium. For cell synchronization at mitosis, cell cultures were incubated with thymidine $(2 \mathrm{mM})$ for $18 \mathrm{~h}$. Subsequently, cells were washed and cultured for an additional $20 \mathrm{~h}$ in fresh medium. Nocodazole $(50 \mathrm{ng} / \mathrm{ml})$ was added to the cultures for the last $16 \mathrm{~h}$. Mitotic cells were collected by shaking off the plates and seeded in complete medium for subsequent analyses. To assess the cell cycle distribution, cells were fixed with chilled $70 \%$ ethanol, stained with $50 \mu \mathrm{g} / \mathrm{ml}$ propidium iodide (PI) and analyzed by flow cytometry (FACSCalibur, BD). To analyze the percentage of cells in mitosis, ethanol-fixed cells were stained with an antibody recognizing Histone $\mathrm{H} 3$ phosphorylated on Serine 10 (p-H3) conjugated with FITC (06-570, Millipore), subsequently incubated with PI and analyzed by flow cytometry. Cell cycle distribution and mitotix index analysis was performed with Summit 4.3 software. For cell proliferation assays, cells were stained with $0.5 \mu \mathrm{M}$ carboxyfluorescein diacetate succinimidyl ester (CFSE) (MolecularProbes) in phosphate buffered saline for $15 \mathrm{~min}$ at $37^{\circ} \mathrm{C}$, washed with complete medium for $20 \mathrm{~min}$ and then treated and cultured as indicated. Cells were fixed for $10 \mathrm{~min}$ in a solution of buffered formaldehyde (3.7\%) and fluorescence was detected and analyzed by flow cytometry. Proliferation Wizard software was used to identify cells in different cellular generations and determine proliferation index, which was calculated as the sum of the cells in all generations including the parental divided by the computed number of original parent cells present at the start of the experiment.

\section{Transfections}

Plasmid transfection was performed using XtremeGENE HD (Roche) transfection reagent following manufacturer's recommendations. Mammalian expression plasmids pRc-CMV-HA-E2F1, pRc-CMV-HA-E2F2, pRc-CMVHA-E2F3, pCEFL-MYC and pFRT/FLAG/HA-DESTLIN28B have been previously described (26-28). For exogenous expression of miRNAs, miRNA genes were expressed in the pMirVec vector (29). To silence endogenous expression of E2F1, E2F2, E2F3, E2F7, c-MYC and LIN28B, and to inhibit endogenous microRNA activity, cells were transfected with commercial siRNAs and with miRVana microRNA Inhibitors, respectively (Life Technologies), at a final concentration of $10 \mathrm{nM}$ using Lipofectamine RNAiMAX (Life Technologies) following manufacturer's recommendations.

\section{RNA expression analyses}

Total RNA extraction was performed with TRIzol Reagent (Life Technologies) and purified using the miRNeasy kit (Qiagen) following the manufacturer's recommendations. For small RNA-Sequencing (RNA-Seq), $2 \mu \mathrm{g}$ of total RNA containing the small RNA fraction including miRNAs was processed using the TruSeq Small RNA Sample Preparation kit from Illumina. The resulting libraries were sequenced on the Genome Analyzer IIx with SBS TruSeq v5 reagents following manufacturer's protocols. To test for differential miRNA expression between different samples the Bioconductor DESeq package was used (30). The list of differentially expressed miRNAs produced by DESeq was further filtered to remove miRNAs with fewer than 10 reads in the different samples under comparison (31). Clustering analysis of differentially expressed miRNAs was performed with Perseus software (http://www. perseus-framework.org/).

Predicted targets of microRNAs were identified using the DIANA-microT-CDS miRNA target prediction server (32) and then analyzed for pathway enrichment using terms from the Reactome database (33). We used low $P$-values ( $P$ 
$<0.001$ ) to avoid inconsistent results due to the use of different databases or algorithms, as suggested previously (34). Gene ontology analysis was performed using the FatiScan algorithm (35).

Mature microRNA and Primary microRNA (PrimiRNA) RT-Q-PCR analyses were performed using specific TaqMan microRNA and Pri-miRNA assays, respectively (Life Technologies) (Supplementary Table $\mathrm{S} 1)$. For mRNA expression analysis, RNA was reversetranscribed into cDNA with the High-Capacity cDNA RT Kit (Life Technologies) and Q-PCR was performed as described previously (36). Sequences of Q-PCR primers are listed in Supplementary Table S2.

\section{Protein expression analyses}

For western blot analyses, cells were lysed in buffer containing $10 \mathrm{mM} \mathrm{NaH} \mathrm{PO}_{4} \mathrm{pH} \quad 7.2 ; 1 \mathrm{mM}$ EDTA; 1 mM EGTA; $150 \mathrm{mM} \mathrm{NaCl} ; 1 \%$ NP-40 and a cocktail of protease and phosphatase inhibitors (Roche). Protein concentrations in supernatants were determined using a commercially available kit (DC Protein Assay from Bio-Rad). A total of $20 \mu \mathrm{g}$ of protein were loaded per lane, fractionated in $8-10 \%$ sodium dodecyl sulphatepolyacrylamide gels and transferred onto nitrocellulose membranes (Bio-Rad). Antibodies against the following proteins were used: E2F7 (sc-32574, Santa Cruz), Cyclin E1 (4129, Cell Signaling), c-MYC (sc-42, Santa Cruz), LIN28B (4192, Cell Signaling), HA (MMS-101R, Covance), p-H3 (06-570, Millipore), $\alpha$-Tubulin (T-9026, Sigma), $\beta$-Actin (A5441, Sigma). Immunocomplexes were visualized with horseradish peroxidase-conjugated anti-mouse, anti-goat or anti-rabbit IgG antibodies (Santa Cruz), followed by chemiluminiscence detection (ECL, Amersham) with a ChemiDoc camera (Bio-Rad).

\section{Chromatin immunoprecipitation}

Chromatin immunoprecipitations (ChIPs) and the quantification of immunoprecipitated DNA sequences by Q-PCR were performed as described previously (36). The localization of E2F motifs in E2F7-regulated miRNAs was carried out with the MotifLocator tool of the TOUCAN program (37). The search was restricted to the proximal promoter region $(-1000$ and +500 bp relative to the transcription start site) (38). Sequences of Q-PCR primers are listed in Supplementary Table S3. Antibodies used for ChIP analysis were: E2F1 (sc-193, Santa Cruz), E2F2 (sc-633, Santa Cruz), E2F3 (sc-878, Santa Cruz), E2F4 (sc-1082, Santa Cruz), E2F7 (sc-66870, Santa Cruz), RB (sc-50 Santa Cruz), p107 (sc-318 Santa Cruz), p130 (sc-317 Santa Cruz), MYC (sc764 Santa Cruz), RNA polymerase II (sc-899, Santa Cruz) and SV40LT (sc-147, Santa Cruz).

\section{Statistical analysis}

Data are presented as mean $\pm \mathrm{SD}$. The significance of the difference between two groups was assessed using the Student two-tailed $t$-test. A $P<0.05$ was considered statistically significant.

\section{RESULTS}

\section{Acute loss of E2F7 accelerates cell cycle progression}

E2F7 gene expression is regulated in a cell cycle-dependent manner in U2OS cells, with reduced levels at M and G1 phases and a peak expression in G1/S transition and $S$ phase (Supplementary Figure S1 A and B), consistent with previous reports (11). We assessed whether E2F7 is required for timely cell cycle progression by acutely depleting E2F7 and examining cell cycle distribution over time. Endogenous E2F7 was depleted very efficiently in U2OS cells individually transfected with three independent RNAi molecules specific for E2F7 (siE2F7), but not in cells transfected with an oligonucleotide whose sequence has no specificity to any human protein (siNT) used as a control (Figure 1A and Supplementary Figure S2). Importantly, E2F7 depletion resulted in substantially increased mRNA levels of known E2F7-downregulated genes (E2F1, E2F2, E2F3 and Cyclin E1), confirming loss of E2F7-mediated repression in siE2F7-transfected cells (Figure 1A and Supplementary Figure S2).

To monitor cell cycle progression upon acute silencing of E2F7, U2OS cells were HU-synchronized at G1/S boundary and subsequently transfected with E2F7-specific siRNAs. Upon removal of the drug, cells were harvested every $3 \mathrm{~h}$ for FACS analyses (Figure 1B). DNA content analyses revealed a comparable block in G1/S in non-target control and E2F7 siRNA transfected cells. Remarkably, upon HU release, entry into S-phase in E2F7-depleted cells was significantly accelerated compared to control cells. This effect was visible as early as $3 \mathrm{~h}$ after $\mathrm{HU}$ release $(48 \%$ in siE2F7 versus $40 \%$ siNT). Likewise, E2F7 depleted cells showed an earlier entry into $\mathrm{G} 2$ phase $6 \mathrm{~h}$ after exiting from HU-induced block (41 versus 27\%) and it was also evident at the $9 \mathrm{~h}$ time point (67 versus 52\%). Similarly, E2F7 depleted cells synchronized in M-phase with nocodazole showed accelerated entry and progression into S phase as well as into G2 (50\% in siE2F7 versus $39 \%$ siNT at the $15 \mathrm{~h}$-time-point following exit from mitotic arrest) in comparison with control siRNA transfected cells (Supplementary Figure S3). Consistent with these results, E2F7 siRNA transfected cells exhibited earlier and increased levels of the mitotic marker $\mathrm{p}$ $\mathrm{H} 3$ after cell cycle re-entry from a HU-induced block (Figure 1C). By contrast, E2F8 depletion in U2OS cells did not result in a significant impact on cell cycle progression, even though E2F7 and E2F8 showed similar expression levels in U2OS cells (Supplementary Figure S4).

In parallel, we examined the rate of cell proliferation by labeling the cells with the vital fluorescent dye CFSE, which is diluted $\sim 2$-fold with each cell division. Consistent with cell cycle analyses, E2F7-depleted cells displayed a higher proliferation rate compared to siNT cells (Figure 1D). Thus, by inducing acute loss of E2F7, our data reveal that E2F7 is indispensable for correct progression through the cell cycle and for cellular proliferation, and that this role is not satisfied by E2F8 or by other E2F family members upon E2F7 knockdown. 


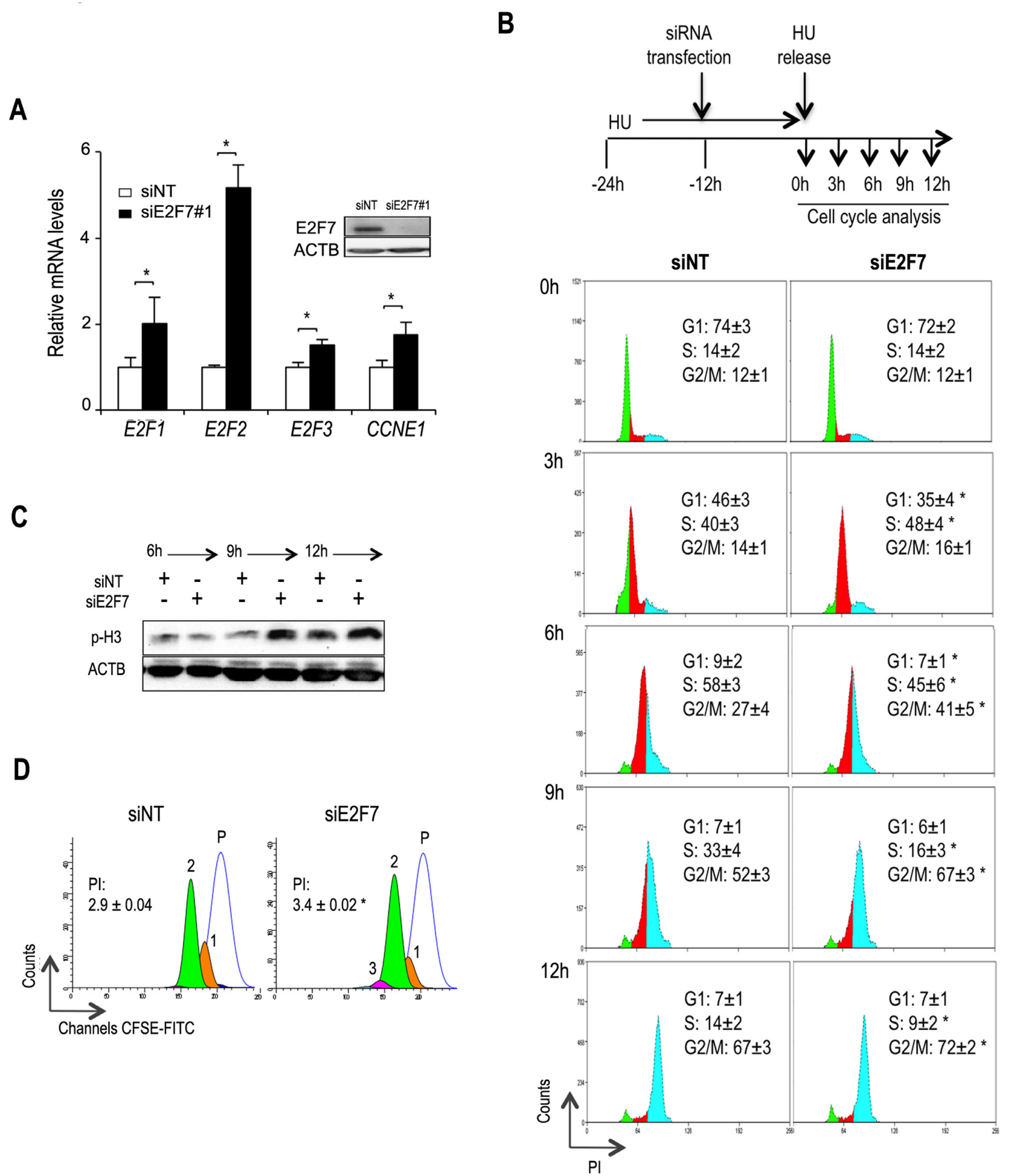

Figure 1. Accelerated cell cycle progression and increased proliferation in cells lacking E2F7. (A) RT-Q-PCR analyses of E2F1,E2F2,E2F3 and Cyclin E1 (CCNE1) in cells treated with siE2F7 \#1 or a non-target siRNA (siNT) for $12 \mathrm{~h}$. Western blot analysis shows efficient depletion of E2F7 by transfection of specific siRNA molecules. mRNA expression values are normalized to the expression of EIF2C2, used as a standard control. Results are expressed as fold over siNT values (mean \pm SD) from three independent experiments. (B) U2OS cells were treated with $4 \mathrm{mM}$ HU and $12 \mathrm{~h}$ later were transfected with NT siRNA and E2F7 siRNA (\#1). Cells were washed after $24 \mathrm{~h}$ of HU treatment, harvested every $3 \mathrm{~h}$ and processed for FACS analysis. Shown is the experimental design followed. The percentage of cells in G1 (green), S (red) and G2/M (blue) \pm SEM (standard error of the mean) is indicated and correspond to the average of three independent replicates. (C) Lysates from cells treated as in panel B, harvested at indicated times after HU release were used for western blot analyses of p-H3 (Ser 10). (D) Proliferation of representative siNT and siE2F7 transfected cultures. U2OS cells were incubated with CFSE, transfected with indicated siRNAs and cultured for $24 \mathrm{~h}$. Proliferation Index (PI) corresponds to the average of three experiments. Shown are representative images of the parental population $(\mathrm{P})$ and the proliferative cellular generations in each condition (indicated with numbers). Asterisks $(*)$ indicate significant differences $(P<0.05)$, and were derived from a two-tailed $t$-test between siE2F7- and siNT-transfected cells. 


\section{Identification of E2F7-regulated miRNAs}

We set out to identify microRNAs that could contribute to E2F7-mediated cell cycle and proliferation control. To this end, unbiased RNA-Seq experiments were conducted using RNA derived from E2F7-competent and E2F7-depleted cells at $\mathrm{G} 1 / \mathrm{S}$ transition $(0 \mathrm{~h}), \mathrm{S}$ phase $(3 \mathrm{~h})$ and $\mathrm{G} 2 / \mathrm{M}$ boundary $(12 \mathrm{~h})$ of cell cycle following exit from HUinduced block. The expression level of endogenous E2F7 was appreciable in all three time-points (Supplementary Figure S1B). Three independent RNA-Seq experiments were performed for each condition (siE2F7 versus siNT) and time-point. Close to 1100 miRNAs were identified in the three time-points analyzed. A list of differentially expressed miRNAs between control and E2F7-depleted cells was produced by Bioconductor DESeq package (30) and only those miRNAs with fold-changes higher than 1.5 (siE2F7 versus siNT) in at least two of the three experiments were considered. Using these criteria a total of $18 \mathrm{miR}$ NAs were found to be consistently deregulated upon E2F7 knockdown (Figure 2A and Supplementary Table S4), 15 of which were upregulated in at least two time-points of the cell cycle. These data suggest a major role for E2F7 as a negative regulator of miRNA expression throughout the cell cycle.

Within the set of E2F7-regulated miRNAs, miR-25, let7f and miR-92a have been previously identified as E2F1 and E2F3 targets (14,17-19,39). Of note, whereas E2F1 and E2F3 are known to induce the expression of these miRNAs, our data indicate that E2F7 represses their expression. In addition, our deep-sequencing analyses produced many other miRNAs that represent potentially novel E2Fregulated miRNAs (Figure 2A and Supplementary Table S4). E2F7 has been reported to repress E2F site-dependent transcription $(7,8,11)$. To identify potential E2F motifs within E2F7-represed microRNAs, we made use of the MotifLocator tool provided by TOUCAN program (37). Using a threshold level of 0.8 for similarity with the canonical E2F motif recorded in the TRANSFAC database, we found that $67 \%$ of E2F7-repressed genes harbored at least one canonical E2F motif within the $-1000 /+500$ bp regulatory region (Supplementary Table S5).

From the collection of miRNAs that were differentially expressed in E2F7-depleted cells, we selected those that have been previously related with E2F (miR-25, let-7f and miR-92a) as well as a set of miRNAs bearing E2F motifs in their promoter regions (let-7b, miR-26a, miR-27b and miR-7) (Supplementary Table S5) for further analyses. Conventional RT-Q-PCR assays of the selected miRNAs showed significantly increased expression levels upon E2F7 knockdown in the three cell cycle phases analyzed (Figure 2B), thus validating the small RNA-Seq experiment results. We subsequently examined potential pathways regulated by these miRNAs by performing a bioinformatics analysis of their predicted targets. Interestingly, Gene Ontology analysis of the combined predicted targets revealed that E2F7repressed miRNAs preferentially modulate genes involved in cell cycle and mitotic regulation (Figure 2C). Other biological processes including hemostasis, signaling by Nerve Growth Factor (NGF) or transmembrane transport also appeared enriched in this analysis, suggesting that E2F7 regulates a diversity of functions through control of microRNA expression.

\section{E2F7-repressed miRNAs modulate cell proliferation}

We tested whether miRNAs repressed by E2F7 (miR25, let-7f, let-7b, miR-26a, miR-27b, miR-92a and miR7) could contribute to E2F7-dependent control of the cell cycle. U2OS cells were transfected with expression vectors coding for these miRNAs, and cell cycle distribution profiles were analyzed. Ectopic expression of individual miRNAs gave rise to a slight acceleration of the first cell division cycle relative to scramble control-transfected cells (Supplementary Figures S5, S6 and data not shown). Importantly, this effect was amplified after several cell division cycles, and we observed significantly increased proliferation rates when fluorescence of CFSE-stained cells was quantified after overexpression of individual miRNAs (Figure 3A and Supplementary Table S6). Furthermore, blocking endogenous miRNA activity with a pool of anti-miRNA oligonucleotides reversed the accelerated cell cycle progression induced by E2F7 knockdown (Supplementary Figure S7).

We next assessed whether E2F7-regulated miRNAs could promote cell proliferation by limiting the expression of miRNA target genes involved in cell growth inhibition. Several critical cell cycle inhibitors reported to be regulated by these miRNAs, such as p21 ${ }^{\text {Cip1 }}$, p5 $7^{\text {Kip2 }}$, PTEN and p130, were indeed downregulated in U2OS cells overexpressing individual E2F7-repressed miRNAs (Figure 3B). p18 $8^{\mathrm{INK} 4 \mathrm{C}}$, which has not been reported to be regulated by microRNAs, showed no differences in this assay, ruling out possible general effects due to an overall proliferation increase. Collectively, these results point to a role for these microRNAs in E2F7-mediated negative regulation of cell proliferation and cell cycle control by modulating the levels of critical cell cycle inhibitors.

\section{E2F factors are bound to the promoter region of miR-25, miR-26a, miR-27b, miR-92a and miR-7}

To begin to dissect the mechanism by which E2F7 represses the expression of miRNAs during the cell cycle, we examined binding of E2F7 to the regulatory regions of the validated miRNAs. Binding of E2F7 was examined by ChIP analyses followed by Q-PCR with specific oligonucleotides for each miRNA regulatory region bearing E2F consensus sites (Figure 4A). The regulatory region of let-7f lacks putative E2F binding sites, and was therefore discarded for binding studies. The $\beta$-actin gene (ACTB), whose promoter lacks active E2F sites (36), was used as a negative promoter control. We made use of chromatin derived from cells collected at $3 \mathrm{~h}$ following HU release (corresponding to S-phase cells). Additionally, as a control for non-specific ChIP, parallel ChIP assays were carried out with an irrelevant antibody (SV40LT). As shown in Figure 4B, ChIP analyses revealed robust E2F7 binding to the regulatory region of miR-25, miR-92a and miR-7 (compare binding to ACTB), suggesting that E2F7 represses miR-25, miR-92a and $\mathrm{miR}-7$ expression by directly binding to their regulatory region. By contrast, we did not to detect binding of E2F7 to the regulatory regions of miR-26a, miR-27b and let-7b, which harbor consensus E2F sites (Figure 4A and B). 
B

\section{A}

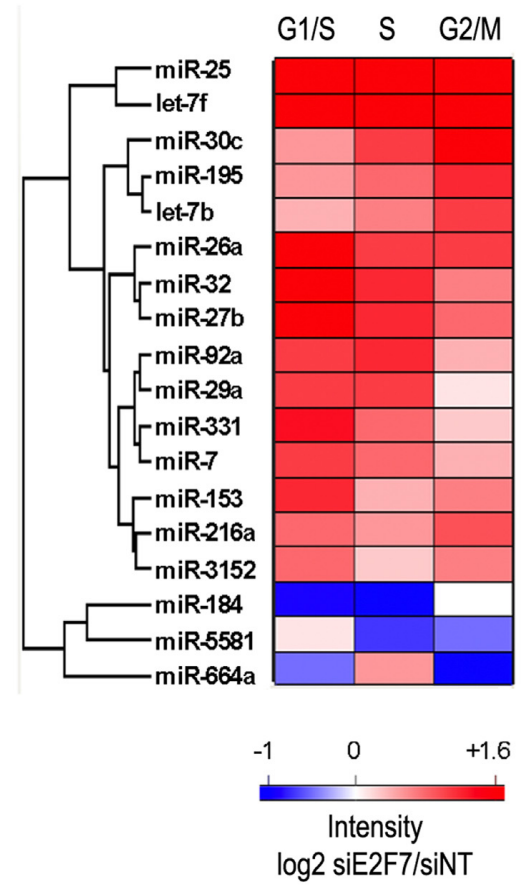

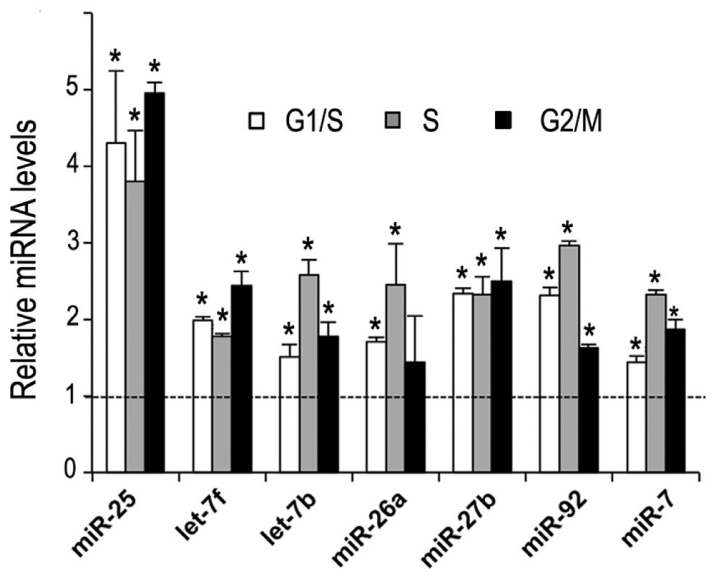

C

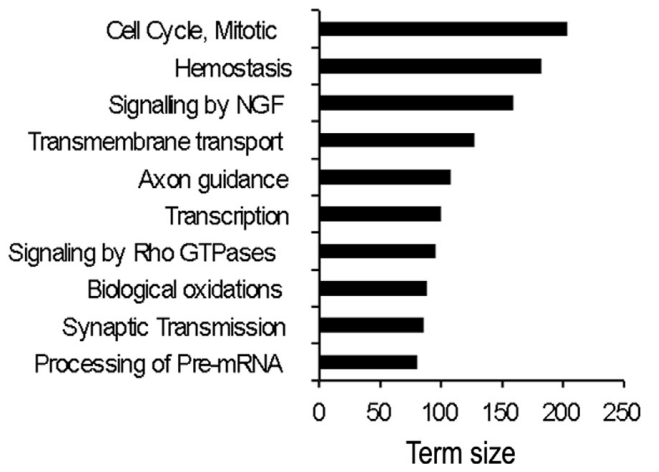

Figure 2. Identification of E2F7-regulated miRNAs by small RNA-Seq analysis. (A) MicroRNA expression profiles in siE2F7\#1 transfected cells at various time-points after HU release. Data are normalized to the levels of siNT-treated cells. Red indicates upregulation and blue indicates downregulation. (B) Confirmatory RT-Q-PCR expression analyses in E2F7-depleted cells at various time-points after HU release. Mature miR-25, let-7f, let-7b, miR-26a, miR-27b, miR-92a and miR-7 expression levels were analyzed and normalized to RNU6B and RNU19 levels. Data are represented as fold-change relative to siNT-transfected samples $(* P<0.05)$. (C) Gene ontology $(\mathrm{GO})$ analysis of predicted targets of E2F7-repressed miRNAs using FatiGO tool. Only terms with adjusted $P$-value of $>0.001$ were considered.

It has been shown that individual E2F target promoters are bound by multiple E2Fs in vivo $(36,40)$. Therefore, we tested whether other E2F family members could occupy the regulatory regions of these miRNAs. We focused on E2F1, E2F2 and E2F3 because they are regulated by E2F7 (Figure 1A), and thus, could potentially be involved in E2F7-dependent miRNA regulation. As shown in Figure $4 \mathrm{C}$, we found that E2F1, E2F2 and E2F3 were efficiently recruited to the promoter region of miR-25 and miR-92a ( $>2$-fold over $\beta$-actin promoter amplification), supporting previous data $(14,18,19)$. Interestingly, miR-26a regulatory region was bound by E2F3, and both, E2F1 and E2F3, occupied miR-27b and miR-7 promoters. None of them was significantly recruited to let-7b. Remarkably, E2F7 depletion led to a dramatic increase in recruitment of E2F1, E2F2 and E2F3 to miR-25, miR-26a, miR-27b, miR-92a and miR-7 promoters (Figure 4C, note scale difference), consistent with the increased expression of these E2Fs in E2F7-knockdown cells (Figure 1A). Moreover, ectopic expression of E2F1-3 factors led to an induction of E2F7regulated miRNAs (Supplementary Figure S8). These results point to a direct role for E2F1, E2F2 and E2F3 in the transcriptional activation of E2F7-repressed miRNAs.

\section{E2F7 regulates let-7 microRNA maturation}

The absence of consensus E2F motifs in let-7f regulatory region and the lack of binding of E2F factors to let-7b, points to an indirect mechanism for E2F7 in the regulation of these miRNAs. We examined the abundance of let$7 \mathrm{f}$ and let-7b immature primary transcripts in U2OS cells transfected with non-target or E2F7-specific siRNAs. We included miR-25 in our assay as a control of a miRNA whose promoter is bound by E2F7 (Figure 4B). Unprocessed pri-miR-25 levels were increased in E2F7-depleted cells (Figure 5A), demonstrating that E2F7 regulates miR25 expression at the transcriptional level. By contrast, prilet-7f and pri-let-7b levels remained unaffected upon E2F7 knockdown. These findings rule out a transcriptional regulation of let-7f and let-7b by E2F7 and point to a role of this E2F factor in the maturation pathway of let-7 miRNAs.

The RNA binding proteins LIN28A and LIN28B have been reported to directly bind to let-7 precursor miRNA molecules and inhibit their processing into mature and functional miRNAs $(41-45)$. We tested whether E2F7 depletion had an effect on LIN28 expression. Interestingly, RT-Q-PCR and western blot analyses showed a substantial reduction of LIN28B mRNA and protein levels (both al- 
A
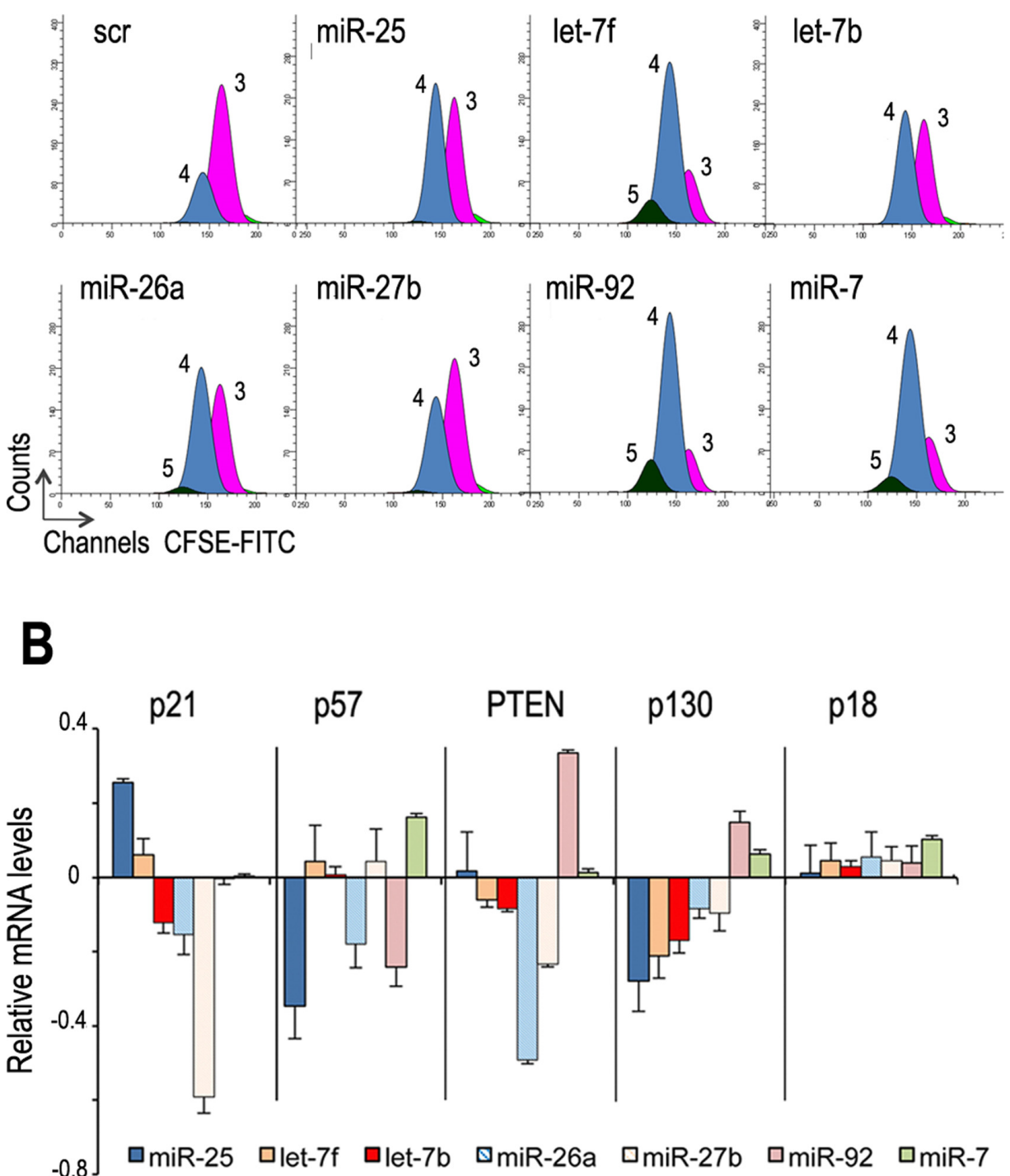

Figure 3. E2F7-regulated miRNAs target critical cell cycle regulators and enhance cell proliferation. (A) U2OS cells were transfected with various miRNAcoding plasmids and incubated with CFSE. A vector coding a scramble sequence (scr) was used as a negative control. Cells were harvested 24,48 and 72 $\mathrm{h}$ after transfection and CFSE fluorescence was determined by flow cytometry. Shown are representative images of the distribution of cellular generations $48 \mathrm{~h}$ after transfection (indicated with numbers). (B) p21 ${ }^{\mathrm{CIP1}}$, p $57^{\mathrm{KIP} 2}$, PTEN, p130 and p18 ${ }^{\mathrm{INK} 4 \mathrm{C}}$ mRNA levels were assessed by RT-Q-PCR in RNA samples extracted from cells treated as in panel A. Data are represented as normalized $\log _{2}$-ratios over control scr transfection.

ternatively spliced forms) upon E2F7 knockdown (Figure 5B). LIN28A levels were not detected in U2OS cells (data not shown).

We next assessed whether LIN28B was required for downregulation of let-7b and let-7f in cell cycle synchronized U2OS cells. Knockdown of LIN28B by RNAi led to an increased expression of endogenous let-7b and let-7f (Figure 5C). Conversely, ectopic LIN28B expression abolished the increased expression levels exhibited by let-7f and let-7b in cells lacking E2F7, but not the levels of miR25 (Figure 5D). Collectively, these results imply a posttranscriptional pathway regulated by E2F7 and LIN28B in dictating the levels of let-7 miRNAs.

\section{E2F7 regulation of let-7 involves the LIN28/c-MYC axis}

LIN28B expression has not been previously linked to E2F. Instead, LIN28B expression is known to be induced by cMYC (46). In addition to the c-MYC binding site previously reported (46), inspection of LIN28B promoter region revealed three putative E2F-recognition sites near the transcription start site (Figure 6A). However, ChIP analyses did not detect endogenous E2F7 bound to LIN28B promoter. Likewise, E2F1, E2F2 and E2F3 were absent from LIN28B promoter both in control- and E2F7-depleted cells (Supplementary Figure S9 and data not shown). In contrast, c-MYC was efficiently recruited to the promoter region of LIN28B (Figure 6A), and subsequent functional analyses showed that knockdown of c-MYC led to decreased 

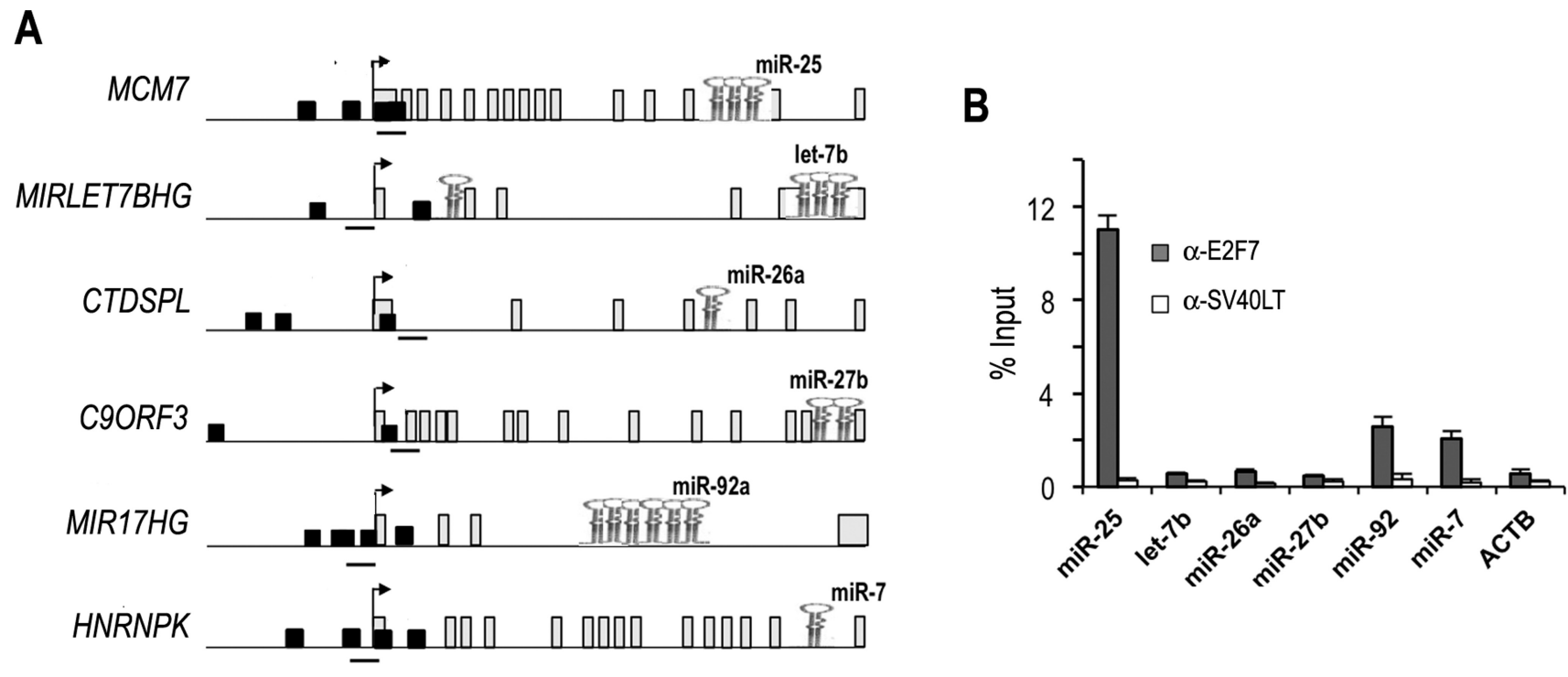

C
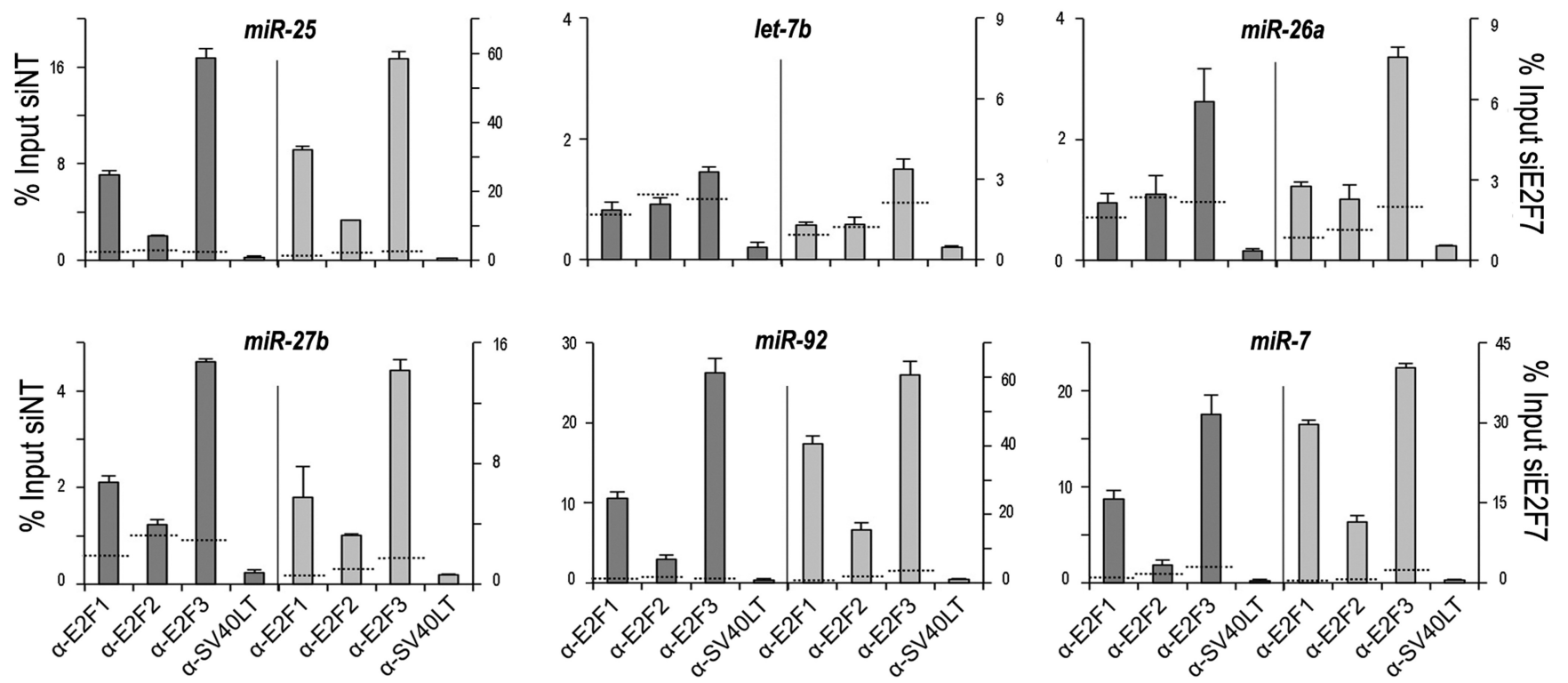

Figure 4. E2F factors are recruited to the promoter region of multiple microRNAs. (A) Schematic representation of human miR-25, let-7b, miR-26a, miR27b, miR-92a and miR-7 loci within the human MCM7, MIRLET7BHG, CTDSPL, C9ORF3, MIR17HG and HRNPK transcription units, respectively. The predicted E2F recognition sites are indicated by small filled boxes. Horizontal lines depict the chromatin sequences amplified by Q-PCR. (B) ChIPQ-PCR analyses of E2F7-regulated miRNAs. Cell lysates were harvested $3 \mathrm{~h}$ after HU release and used for ChIP assays with an antibody against E2F7. Promoter regions near E2F consensus sites were amplified by Q-PCR. The promoter of $\beta$-Actin $(A C T B)$ was used as a negative control. An unrelated antibody against the SV40 large T antigen (SV40LT) was used as a control for background immunoprecipitation. Data are presented as percentage of input chromatin (representative experiment of three independent experiments where the values are the mean $\pm \mathrm{SD}$ of triplicate determinations). (C) Cell lysates from siNT and siE2F7-transfected cells were harvested $3 \mathrm{~h}$ after HU release and used for ChIP assays with antibodies against E2F1, E2F2 and E2F3. Note the Y-axis scale difference in the siE2F7-treated samples. ACTB amplification values are represented as dotted horizontal lines.

LIN28B mRNA and protein levels (Figure 6B), suggesting that c-MYC directly transactivates LIN28B expression in U2OS cells.

Importantly, ectopic expression of c-MYC impaired the reduction of LIN28B expression in E2F7-depleted cells, and led to a recovery of LIN28B levels close to those in siNT-treated cells (Figure 6C), suggesting that E2F7 could control LIN28B expression indirectly through the modula- tion of c-MYC levels. Furthermore, let-7b upregulation in siE2F7 treated cells was partially reversed upon c-MYC expression, whereas miR-25 expression was not negatively affected by c-MYC (Figure 6D).

The above results raised the possibility that E2F7 may control let-7 and LIN28B expression through c-MYC. Indeed, we found that c-MYC expression was significantly decreased upon knockdown of endogenous E2F7 by two inde- 
A

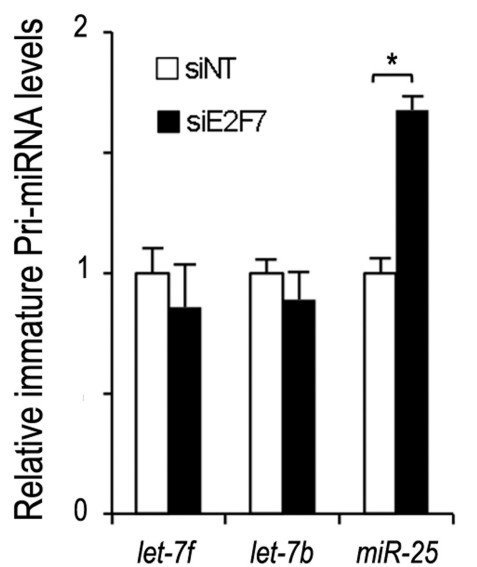

B

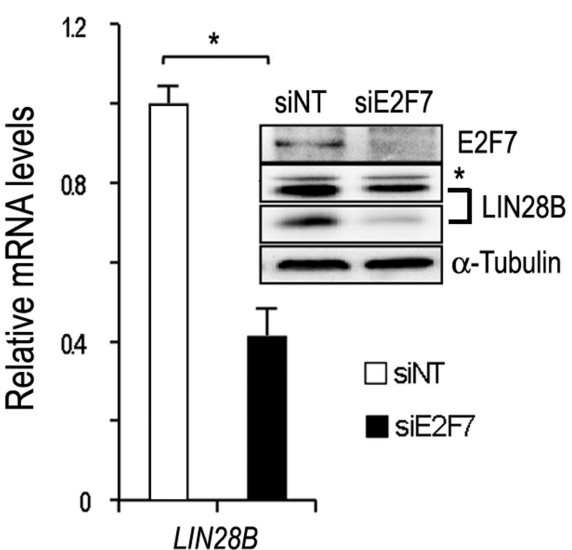

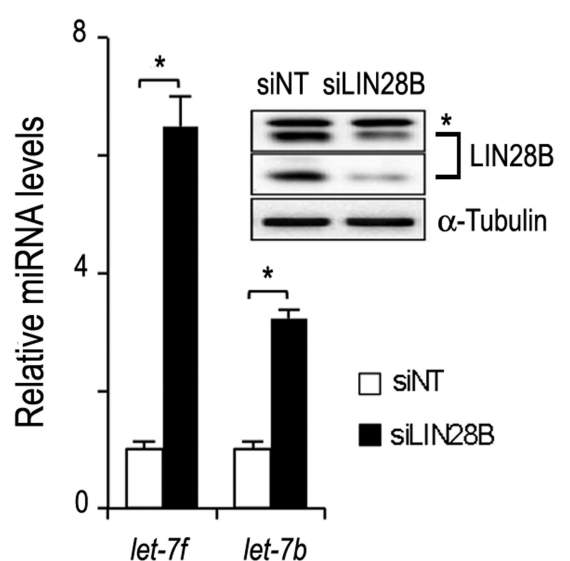

let-7f let-7b

\section{D}

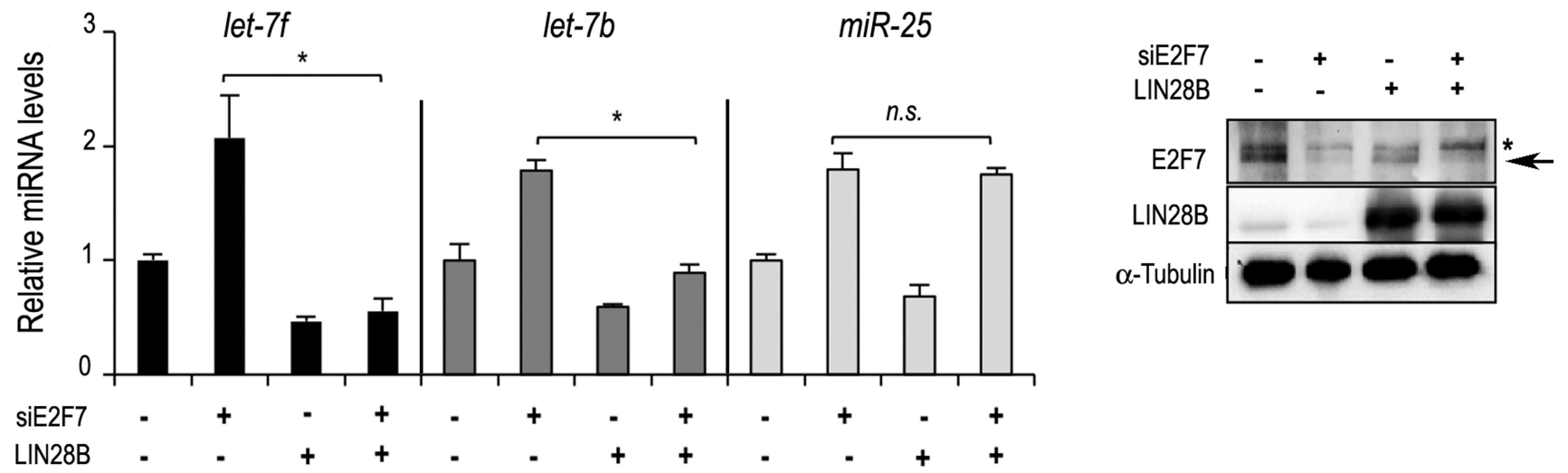

Figure 5. E2F7 controls let-7f and let-7b maturation through LIN28B (A) E2F7 does not regulate let-7f and let-7b transcription. HU-synchronized cells were transfected with siNT and siE2F7 and RNA was purified $3 \mathrm{~h}$ after cell cycle re-entry. Expression analyses of the indicated pri-miRNAs were performed using specific Taqman assays. (B) LIN28B expression was analyzed by RT-Q-PCR in cells transfected with siNT or siE2F7 RNAs. mRNA expression values are normalized to the expression of EIF2C2, used as a standard control. Western blot analysis shows significant downregulation of LIN28B expression after E2F7 depletion. Specific bands corresponding to two isoforms of LIN28B are indicated with arrows. A non-specific band is indicated with an asterisk. (C) LIN28B controls let-7f and let-7b expression in U2OS cells. U2OS cells were synchronized in mitosis by nocodazole treatment and transfected with LIN28B siRNA molecules. RT-Q-PCR analyses of let-7f and let-7b were carried out with RNA samples after $6 \mathrm{~h}$ of mitotic block release. Let-7f and let-7b expression was normalized to RNU6B and RNU19 small RNAs expression, used as standard controls. Data are represented as fold change relative to siNT. Western blot shows efficient siRNA-mediated knockdown of LIN28B. (D) E2F7 and LIN28B cooperate to regulate let-7f and let-7b expression. let-7f, let-7b and miR-25 levels were determined in U2OS cells synchronized in mitosis and transfected with E2F7 siRNA together with an expression plasmid encoding LIN28B (pFRT-LIN28B). Cells were harvested $6 \mathrm{~h}$ after block release. miRNA expression levels are shown over the empty transfection. $\left({ }^{*} P<\right.$ 0.05 in all graphs). Western blot shows expression of E2F7 and LIN28B in the samples used for miRNA expression analysis. A non-specific band in E2F7 blot is indicated with an asterisk.

pendent siRNA molecules (Figure 6E and Supplementary Figure S10). This surprising result prompted us to examine c-MYC regulation in more detail. c-MYC promoter region has been reported to contain several E2F elements (47), and our bioinformatic analysis confirmed this point (Figure 7A). However, whether E2Fs are involved in c-MYC regulation has not been clarified. We assessed c-MYC promoter occupancy by E2F7 as well as by its targets E2F1, E2F2 and E2F3. ChIP analyses revealed robust binding by all four E2Fs to c-MYC promoter. In addition, E2F7-depleted cells exhibited dramatically increased promoter binding by E2F1, E2F2 and E2F3 factors (Figure 7A, note scale difference), suggesting that E2F7-dependent c-MYC regulation might be mediated, at least in part, by E2F1-3. Ac- cordingly, c-MYC mRNA and protein levels were increased upon E2F1, E2F2 and E2F3 depletion (Figure 7B and Supplementary Figure S11A). By contrast, another E2F target (Cyclin E1) showed decreased expression upon the combined knockdown of E2F1-3 (Figure 7B), as previously reported (48). Moreover, c-MYC expression was negatively affected by the ectopic expression of E2F1-3, both at the mRNA and protein levels (Figure 7C and Supplementary Figure S11B).

Modulation of c-MYC levels by E2F1-3 was not the result of altered cell-cycle profiles in these cells (Supplementary Figure S11C) and it did not involve increased promoter binding by classical E2F repressors, such as E2F4, or by pocket proteins (Supplementary Figure S12 and data 


\section{A}
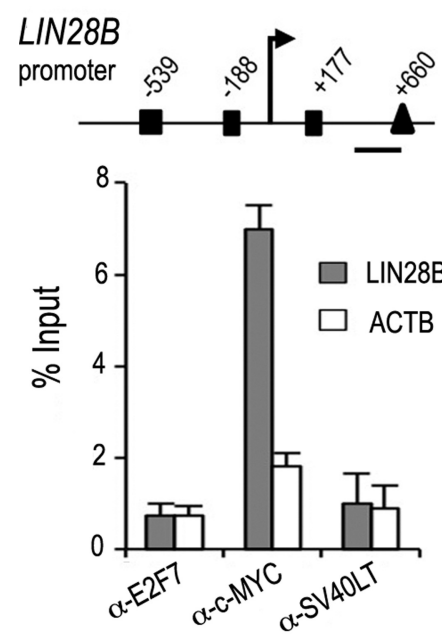

B

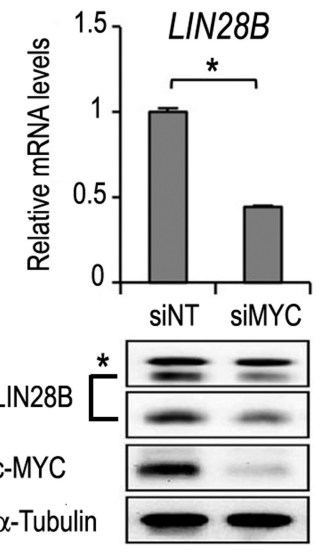

C

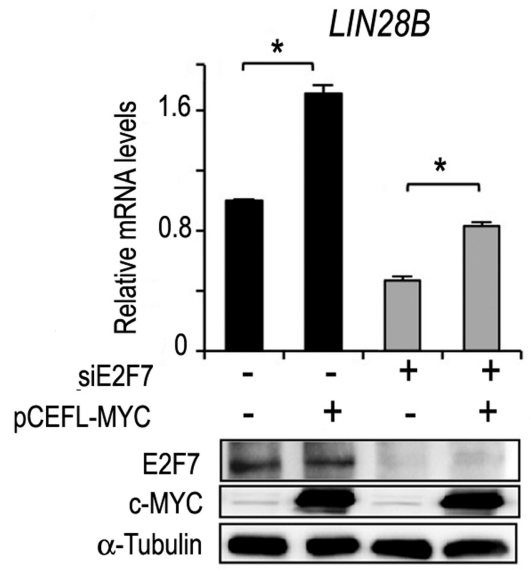

E
D

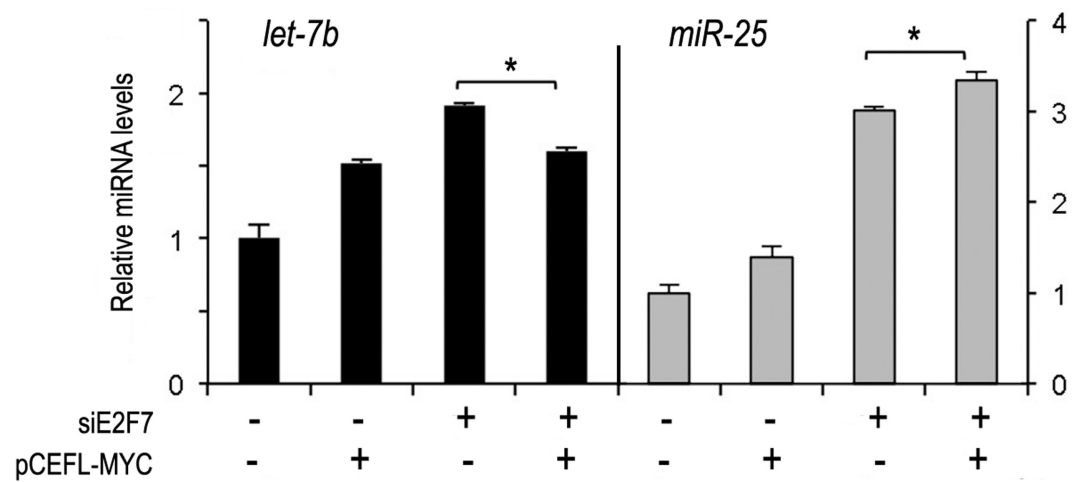

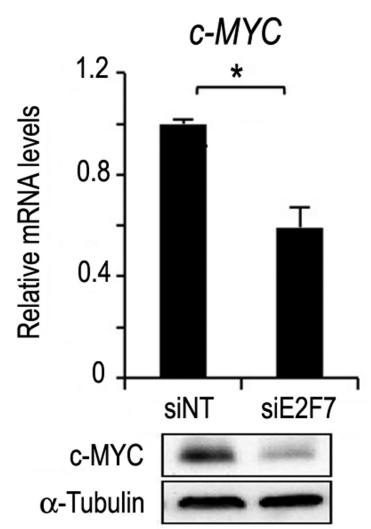

Figure 6. E2F7 controls LIN28B expression through c-MYC. (A) Schematic representation of LIN28B promoter region, indicating the localization of consensus E2F (boxes; -539: TTTCGGGC; - 188: GCACGAAA; +177: TTTGGAGC) and c-MYC (triangle; TCCTCGTGCCC) binding motifs. ChIP analyses were performed with the indicated antibodies and Q-PCR was performed using primers spanning genomic regions around or close to E2F and cMYC sites. The horizontal line depicts the chromatin sequence amplified by Q-PCR. Data correspond to a representative experiment of three independent replicates. (B) RT-Q-PCR and western blot analyses of LIN28B levels in c-MYC depleted cells. Cells blocked in mitosis were transfected with siNT or siMYC molecules and RNA and protein extracts were harvested $9 \mathrm{~h}$ after block release. Western blot shows efficient knockdown of c-MYC in U2OS cells. mRNA data are shown as fold-change over siNT. (C) RT-Q-PCR analysis of LIN28B mRNA levels in U2OS cells synchronized in mitosis and transfected with E2F7 siRNA along with an expression plasmid encoding c-MYC (pCEFL-MYC). Cells were harvested $9 \mathrm{~h}$ after block release. Data are shown as fold over the empty vector transfection. Western blot shows c-MYC and E2F7 expression levels in samples used for LIN28B expression analysis. (D) miRNA levels were assessed in cells treated as in panel C. (E) c-MYC mRNA and protein levels were analyzed in cells synchronized in the cell cycle by HU treatment and transfected with siNT or siE2F7. mRNA expression values are normalized to the expression of $E I F 2 C 2$, used as a standard control. $(* P<0.05)$.

not shown), suggesting that repression of c-MYC by E2F13 could involve RB-independent mechanisms. Consistent with this, a similar level of c-MYC repression by E2F1-3 was detected in HEK293T cells (Supplementary Figure S13), which harbor inactive RB (49).

Importantly, modulation of E2F1-3 levels affected RNA Pol II occupancy downstream of c-MYC transcription start site (Figure 7D), which is a measure of c-MYC transcription rate (50). ChIP data revealed increased association of RNA Pol II with c-MYC gene in E2F1-3 depleted cells, whereas RNA Pol II occupancy in Cyclin E1 gene was decreased in the same experiment. Conversely, ectopic E2F2 expression negatively impacted on RNA Pol II association to c-MYC promoter, while Cyclin E1 gene showed an increased occu- pancy by RNA Pol II in these samples. These results further demonstrate that E2F1-3 repress c-MYC at the transcriptional level. Thus, we conclude that E2F7 regulates let-7 miRNA expression through a miRNA maturation pathway involving several intermediate steps controlled by the transcriptional activity of E2F and c-MYC factors.

\section{DISCUSSION}

In this work, we have analyzed the contribution of E2F7 transcription factor to the regulation of a subset of novel target miRNAs during the cell division cycle. We have discovered transcriptional and post-transcriptional mechanisms by which E2F7 modulates target miRNA expression. Our data support a model whereby E2F7 ensures repression 
A
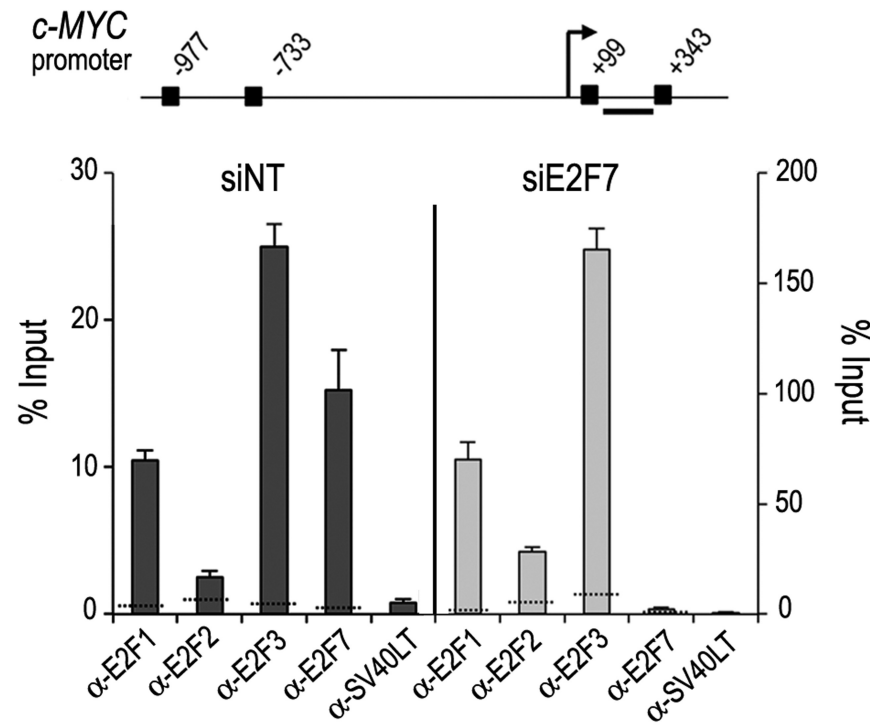

B

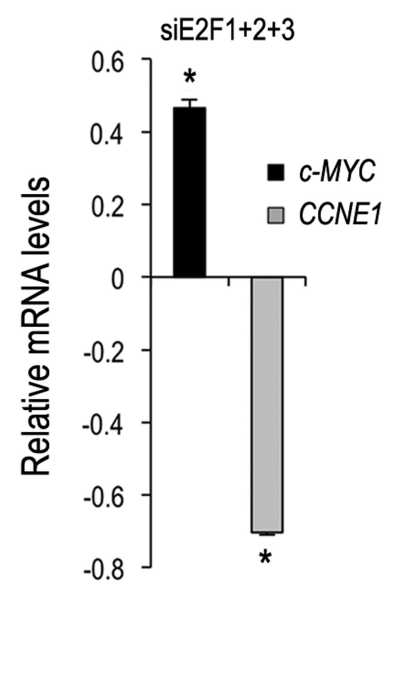

C

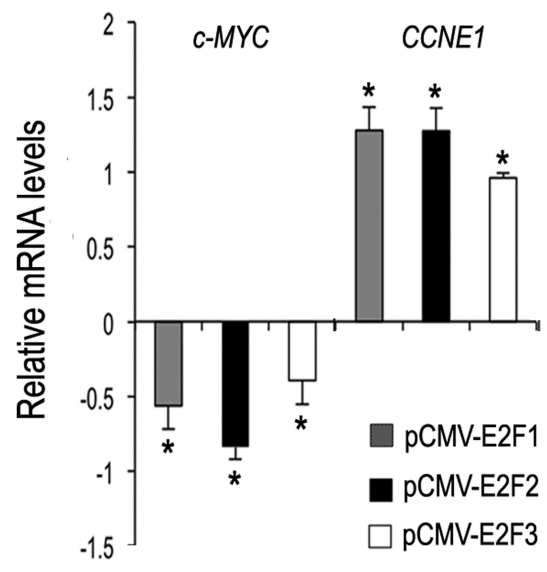

D

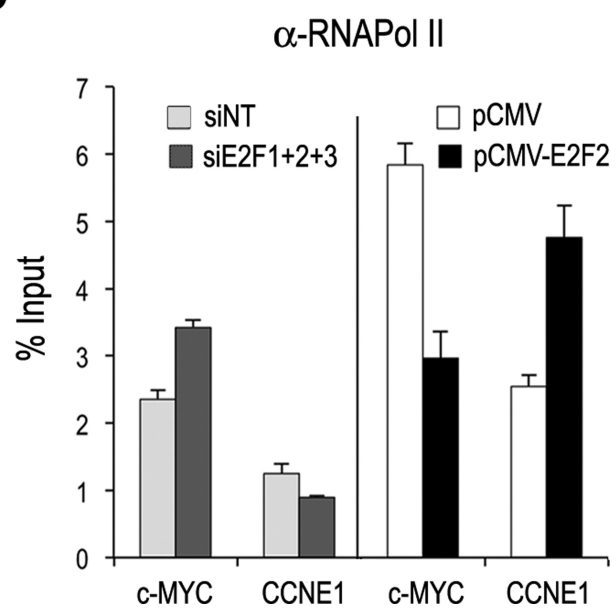

Figure 7. E2F1, E2F2, E2F3 and E2F7 bind to c-MYC promoter and regulate its expression. (A) Schematic representation of c-MYC promoter region, indicating the localization of consensus E2F motifs (filled boxes; -977: GCGCCACA; -733: GCAGCAAA; +99: GCGGGAAA; +343: CTTGCCGC). The horizontal line depicts the ChIP-Q-PCR amplicon. Binding of E2F1, E2F2, E2F3 and E2F7 was assessed by ChIP-Q-PCR in HU-synchronized cells transfected with E2F7 siRNA or control NT siRNAs. Cell lysates of siNT and siE2F7 treated cells were harvested $3 \mathrm{~h}$ after HU release. Note scale difference between siNT and siE2F7. Dotted horizontal lines represent $A C T B$ amplification values. Data correspond to a representative experiment of three independent replicates. (B) c-MYC and CCNE1 expression was analyzed by RT-Q-PCR in cells transfected with siNT or a pool of E2F1, E2F2 and E2F3 specific siRNAs. mRNA data are presented as normalized log2-ratios over siNT transfection. (C) U2OS cells were synchronized in mitosis and transfected with plasmids expressing HA-tagged E2F1, E2F2 and E2F3, $c$-MYC and CCNE1 levels were analyzed by RT-Q-PCR in samples harvested 6 $\mathrm{h}$ after block release. mRNA data are shown as log2-ratios over the empty pCMV transfection. (D) ChIP analyses of RNA-Pol II binding at downstream regions ( $>1 \mathrm{~kb}$ ) of $c-M Y C$ and $C C N E 1$ genes. U2OS cells transfected with E2F1, E2F2 and E2F3 specific siRNAs or pCMV-E2F2-HA were used for ChIP assays with an antibody against RNA-Pol II. Immunoprecipitated DNA was analyzed by Q-PCR using primers in $c-M Y C$ and $C C N E 1+1 \mathrm{~kb}$ region. Data correspond to a representative experiment of three independent replicates.

of a set of miRNA genes throughout the cell cycle, which in turn may finely tune pathways controlling cell proliferation.

The role of E2F7 in cell cycle progression has not been clearly established. Early overexpression experiments suggested that E2F7 could be a negative regulator of the cell cycle $(7,8)$. However, chronic ablation of E2F7 did not impact cellular proliferation (12). By inducing acute depletion of E2F7, and thus largely avoiding compensatory mechanisms that are common after chronic ablation, our data clearly establish a unique requirement for E2F7 in dictating proper cell cycle kinetics, a role that is not shared with E2F8 in U2OS cells. Our observation that E2F7 restrains cell cycle progression raises the possibility that E2F7 could function as a tumor suppressor gene, and is consistent with recent data showing that E2F7 loss together with RB inactivation promotes oncogenic transformation of murine cells (51).

E2F7 has been shown to repress a set of protein-coding genes involved in DNA replication and metabolism (11). The miRNA expression profiling analysis performed in the present work significantly expands our understanding of 
E2F7 function, by providing evidence that this factor has a major role as a negative regulator of miRNA expression. miR-25 and let-7f exhibited the highest levels of overexpression upon E2F7 loss. Interestingly, these miRNAs have previously been identified as induced by E2F1 and E2F3 in S phase entry (14), indicating that E2F7 might repress the expression of miRNAs activated by canonical E2Fs. Other miRNAs previously related to E2F, such as miR-449a/b and miR-15 $(15,16,20)$, were not detected in our work, perhaps because of the restrictive criteria that we used in the different steps of RNA-Seq data analysis. On the other hand, our small RNA-Seq experiment has revealed many other differentially expressed miRNAs that have not been previously linked to E2F activity, and thus represent potentially novel E2F-regulated miRNAs.

Several of the miRNAs that we found to be repressed by E2F7 have previously been described as regulators of proliferation pathways $(13,14,17,23,24,52-59)$. However, their potential roles in cancer have not been clearly established, as these miRNAs appear to have both oncogenic and antioncogenic functions in different cellular contexts. Our data evidence that miR-25, let-7f, miR-27b, let-7b, miR-92a, miR-7 and to a lesser extent miR-26a, promote cell proliferation in U2OS cells, at least in part by silencing the expression of anti-proliferative cell cycle regulators. Thus, by downregulating miRNA expression, E2F7 would indirectly upregulate the levels of cell cycle inhibitors to restrain cell cycle progression. These findings suggest that E2F7 controls cell cycle progression and cellular proliferation through a coordinated performance of both protein-coding and noncoding genes.

According to our promoter occupancy analyses, the chromatin binding properties of E2F7 and canonical E2F factors in the regulation of target genes appear to be different. The finding that E2F7 is only bound to E2F sites present in miR-25, miR-92a, miR-7 and c-MYC, suggests that the affinity of E2F7 for its binding site could be more restricted than that of canonical E2F1-3 factors. The basis for this selectivity remains unknown, and could involve unique interactions between E2F7 and other transcription factors at a particular promoter, as it has been proposed for other members of the E2F family (60).

miRNA biogenesis is thought to be regulated at multiple levels through mechanisms that are still not well understood. Our work reveals that regulation of let-7f and let-7b maturation by E2F7 involves both transcriptional and post-transcriptional mechanisms mediated by c-MYC and LIN28B, adding a new level of complexity to E2Fmediated miRNA regulation. The mechanism by which E2F7 modulates c-MYC expression is presently unknown, although our findings suggest that it involves negative regulation of E2F1-3 and RNA Pol II activities. Interestingly, an interplay between E2F7 and c-MYC activity has recently been suggested (61). Interfering with E2F7 expression resulted in inhibition of c-MYC functional activity in acute myeloid leukemia (AML) cells by an unknown mechanism. However, the authors did not report changes in c-MYC or LIN28B expression. It would be interesting to examine if E2F7 regulates c-MYC gene expression in AML cells, similarly to what we have observed in U2OS cells, and inquire

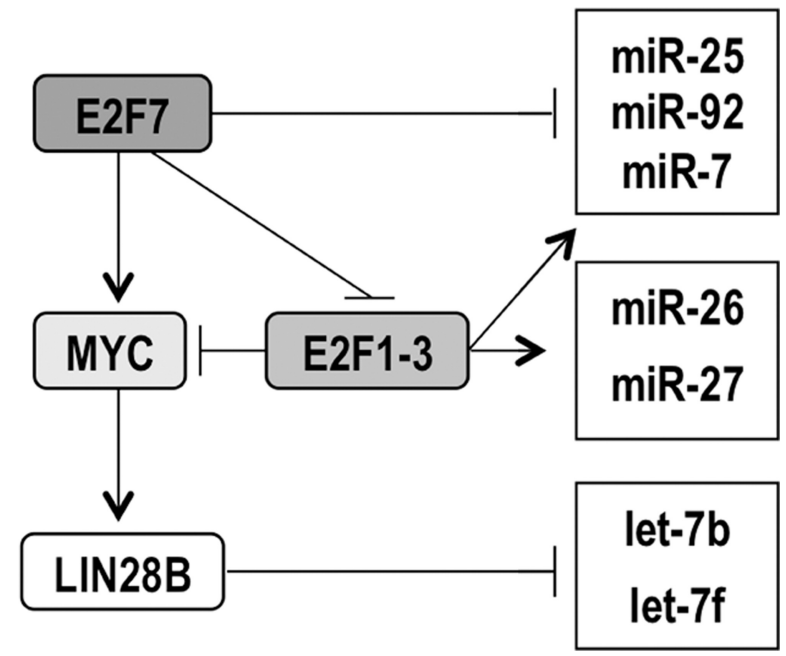

Figure 8. Model summarizing the mechanism of action of E2F factors, c-MYC and LIN28B in miRNA expression regulation described in this study.

into the biological relevance of this novel E2F7-c-MYCLIN28B axis in AML cells.

Overall, our study identifies E2F7 as a critical regulator of miRNA biogenesis throughout the cell cycle (Figure $8)$. Interestingly, we have uncovered a novel interplay between E2F7 and E2F1-3 in the regulation of miRNAs to ensure induction and repression of miRNA genes during the cell division cycle, which in turn could contribute to cell growth control. In this regard, E2F7 might repress miRNA gene expression through multiple mechanisms: by binding to its target genes and directly repressing their transcription (miR-25, miR-92a and miR-7); by repressing the expression of E2F1-3, and indirectly suppressing miRNA expression at the level of transcription (miR-25, miR-26a, miR-27b) or maturation (let-7b, let-7f); or probably by a combination of both mechanisms. These findings support a model in which the transcriptional activity of E2F-target miRNAs may be dictated by an 'E2F-network' in which E2F1-3 and E2F7 play antagonistic roles. A similar mechanism may also be operating in E2F7-mediated regulation of protein-coding genes. Future studies may help to further identify the components of this novel molecular network as well as its biological relevance.

\section{SUPPLEMENTARY DATA}

Supplementary Data are available at NAR Online.

\section{ACKNOWLEDGEMENT}

We thank members of the Zubiaga and Malumbres laboratory for helpful discussions, Naiara Zorrilla for technical support, Ainhoa Iglesias and Asier Fullaondo for assistance with figures, Jose Antonio Rodriguez and Nerea Osinalde for critical reading of the manuscript and J.R. Nevins, $\mathrm{T}$. Tuschl and J. León for kindly providing vectors. 


\section{FUNDING}

This work was supported by the Spanish Ministry [SAF2012-33551, co-funded by the European Regional Development fund to A.M.Z., SAF2012-38215 to M.M., SAF2014-57791-REDC to A.M.Z. and to M.M.]; Basque Government [IT634-13 to A.M.Z.]; University of the Basque Country UPV/EHU [UFI11/20 to A.M.Z.]; Excellence Network CellSYS [BFU2014-52125-REDT to M.M.]; Comunidad de Madrid [S2010/BMD-2470 to M.M.]; Basque Government Fellowship for graduate studies (to J.M.). Funding for open access charge: Basque Government [IT634-13].

Conflict of interest statement. None declared.

\section{REFERENCES}

1. Bertoli,C., Skotheim,J.M. and de Bruin,R.A. (2013) Control of cell cycle transcription during G1 and S phases. Nat. Rev. Mol. Cell Biol., 14, 518-528.

2. Chen,H.Z., Tsai,S.Y. and Leone,G. (2009) Emerging roles of E2Fs in cancer: an exit from cell cycle control. Nat. Rev. Cancer, 9, 785-797.

3. Dimova,D.K. and Dyson,N.J. (2005) The E2F transcriptional network: old acquaintances with new faces. Oncogene, 24, 2810-2826.

4. van den Heuvel,S and Dyson, N.J. (2008) Conserved functions of the pRB and E2F families. Nat. Rev. Mol. Cell Biol., 9, 713-724.

5. Lammens,T., Li,J., Leone,G. and De Veylder,L. (2009) Atypical E2Fs: new players in the E2F transcription factor family. Trends Cell Biol. 19, 111-118.

6. Christensen,J., Cloos,P., Toftegaard,U., Klinkenberg,D., Bracken,A.P., Trinh,E., Heeran,M., Di Stefano,L. and Helin,K (2005) Characterization of E2F8, a novel E2F-like cell-cycle regulated repressor of E2F-activated transcription. Nucleic Acids Res., 33, $5458-5470$

7. de Bruin,A., Maiti,B., Jakoi,L., Timmers,C., Buerki,R. and Leone,G. (2003) Identification and characterization of E2F7, a novel mammalian E2F family member capable of blocking cellular proliferation. J. Biol. Chem., 278, 42041-42049.

8. Di Stefano,L., Jensen,M.R. and Helin,K. (2003) E2F7, a novel E2F featuring DP-independent repression of a subset of E2F-regulated genes. EMBO J., 22, 6289-6298.

9. Logan,N., Graham,A., Zhao,X., Fisher,R., Maiti,B., Leone,G. and La Thangue,N.B. (2005) E2F-8: an E2F family member with a similar organization of DNA-binding domains to E2F-7. Oncogene, 24, 5000-5004.

10. Maiti,B., Li,J., de Bruin,A., Gordon,F., Timmers,C., Opavsky,R., Patil,K., Tuttle,J., Cleghorn,W. and Leone,G. (2005) Cloning and characterization of mouse E2F8, a novel mammalian E2F family member capable of blocking cellular proliferation. J. Biol. Chem., 280, 18211-18220.

11. Westendorp,B., Mokry,M., Groot Koerkamp,M.J., Holstege,F.C., Cuppen,E. and de Bruin,A. (2012) E2F7 represses a network of oscillating cell cycle genes to control S-phase progression. Nucleic Acids Res. 40, 3511-3523.

12. Li,J., Ran,C., Li,E., Gordon,F., Comstock,G., Siddiqui,H., Cleghorn,W., Chen,H.Z., Kornacker,K., Liu,C.G. et al. (2008) Synergistic function of E2F7 and E2F8 is essential for cell survival and embryonic development. Dev. Cell, 14, 62-75.

13. Brosh,R., Shalgi,R., Liran,A., Landan,G., Korotayev,K., Nguyen,G.H., Enerly,E., Johnsen,H., Buganim, Y., Solomon,H. et al. (2008) p53-Repressed miRNAs are involved with E2F in a feed-forward loop promoting proliferation. Mol. Syst. Biol., 4, 229.

14. Bueno,M.J., Gomez de,C.M., Laresgoiti,U., Fernandez-Piqueras,J., Zubiaga,A.M. and Malumbres,M. (2010) Multiple E2F-induced microRNAs prevent replicative stress in response to mitogenic signaling. Mol. Cell. Biol., 30, 2983-2995.

15. Lize,M., Pilarski,S. and Dobbelstein,M. (2010) E2F1-inducible microRNA 449a/b suppresses cell proliferation and promotes apoptosis. Cell Death. Differ., 17, 452-458.
16. Ofir,M., Hacohen,D. and Ginsberg,D. (2011) MiR-15 and miR-16 are direct transcriptional targets of E2F1 that limit E2F-induced proliferation by targeting cyclin E. Mol. Cancer Res., 9, 440-447.

17. Petrocca,F., Visone,R., Onelli,M.R., Shah,M.H., Nicoloso,M.S., De Martino,I., Iliopoulos,D., Pilozzi,E., Liu,C.G., Negrini,M. et al. (2008) E2F1-regulated microRNAs impair TGFbeta-dependent cell-cycle arrest and apoptosis in gastric cancer. Cancer Cell, $\mathbf{1 3}$ 272-286.

18. Sylvestre,Y., De Guire,V., Querido,E., Mukhopadhyay,U.K., Bourdeau,V., Major,F., Ferbeyre,G. and Chartrand,P. (2007) An E2F/miR-20a autoregulatory feedback loop. J. Biol. Chem., 282, 2135-2143.

19. Woods,K., Thomson,J.M. and Hammond,S.M. (2007) Direct regulation of an oncogenic micro-RNA cluster by E2F transcription factors. J. Biol. Chem., 282, 2130-2134.

20. Yang,X., Feng,M., Jiang,X., Wu,Z., Li,Z., Aau,M. and Yu,Q. (2009) miR-449a and miR-449b are direct transcriptional targets of E2F1 and negatively regulate $\mathrm{pRb}-\mathrm{E} 2 \mathrm{~F} 1$ activity through a feedback loop by targeting CDK6 and CDC25A. Genes Dev., 23, 2388-2393.

21. Bueno,M.J., Perez de Castro,I. and Malumbres,M. (2008) Control of cell proliferation pathways by microRNAs. Cell Cycle, 7, 3143-3148.

22. Bueno,M.J. and Malumbres,M. (2011) MicroRNAs and the cell cycle. Biochim. Biophys. Acta, 1812, 592-601.

23. Kim,Y.K., Yu,J., Han,T.S., Park,S.Y., Namkoong,B., Kim,D.H., Hur,K., Yoo,M.W., Lee,H.J., Yang,H.K. et al. (2009) Functional links between clustered microRNAs: suppression of cell-cycle inhibitors by microRNA clusters in gastric cancer. Nucleic Acids Res., 37, 1672-1681.

24. Poliseno,L., Salmena,L., Riccardi,L., Fornari,A., Song,M.S., Hobbs,R.M., Sportoletti,P., Varmeh,S., Egia,A., Fedele,G. et al. (2010) Identification of the miR-106b 25 microRNA cluster as a proto-oncogenic PTEN-targeting intron that cooperates with its host gene MCM7 in transformation. Sci. Signal., 3, ra29.

25. Ventura,A., Young,A.G., Winslow,M.M., Lintault,L., Meissner,A., Erkeland,S.J., Newman,J., Bronson,R.T., Crowley,D., Stone,J.R. et al. (2008) Targeted deletion reveals essential and overlapping functions of the miR-17-92 family of miRNA clusters. Cell, 132, 875-86.

26. Hafner,M., Max,K.E., Bandaru,P., Morozov,P., Gerstberger,S., Brown,M., Molina,H. and Tuschl,T. (2013) Identification of mRNAs bound and regulated by human LIN28 proteins and molecular requirements for RNA recognition. RNA, 19, 613-626.

27. Krek,W., Livingston,D.M. and Shirodkar,S. (1993) Binding to DNA and the retinoblastoma gene product promoted by complex formation of different E2F family members. Science, 262, 1557-1560.

28. Mauleon,I., Lombard,M.N., Muñoz-Alonso,M.J., Cañelles,M. and Leon,J. (2004) Kinetics of myc-max-mad gene expression during hepatocyte proliferation in vivo: Differential regulation of mad family and stress-mediated induction of c-myc. Mol. Carcinog., 39, 85-90.

29. Voorhoeve,P.M., le Sage,C., Schrier,M., Gillis,A.J., Stoop,H., Nagel,R., Liu, Y.P., van Duijse,J., Drost,J., Griekspoor,A. et al. (2007) A genetic screen implicates miRNA-372 and miRNA-373 as oncogenes in testicular germ cell tumors. Adv. Exp. Med. Biol., 604, $17-46$.

30. Anders,S. and Huber,W. (2010) Differential expression analysis for sequence count data. Genome Biol., 11, R106.

31. Dhahbi,J.M., Atamna,H., Boffelli,D., Magis,W., Spindler,S.R. and Martin,D.I. (2011) Deep sequencing reveals novel microRNAs and regulation of microRNA expression during cell senescence. $P L o S$ One, 6, e20509.

32. Paraskevopoulou,M.D., Georgakilas,G., Kostoulas,N., Vlachos,I.S., Vergoulis,T., Reczko,M., Filippidis,C., Dalamagas,T. and Hatzigeorgiou,A.G. (2013) DIANA-microT web server v5.0: service integration into miRNA functional analysis workflows. Nucleic Acids Res., 41, 169-173

33. Joshi-Tope,G., Gillespie,M., Vastrik,I., D’Eustachio,P., Schmidt,E., de Bono,B., Jassal,B., Gopinath,G.R., Wu,G.R., Matthews,L. et al. (2005) Reactome: a knowledgebase of biological pathways. Nucleic Acids Res. 1, 428-432.

34. Ritchie,W., Flamant,S. and Rasko,J.E. (2009) Predicting microRNA targets and functions: traps for the unwary. Nat. Methods, 6, 397-398.

35. Al-Shahrour,F., Minguez,P., Vaquerizas,J.M., Conde,L. and Dopazo,J.2005. BABELOMICS: a suite of web tools for functional annotation and analysis of groups of genes in high-throughput experiments. Nucleic Acids Res., 33, W460-W464. 
36. Infante,A., Laresgoiti,U., Fernandez-Rueda,J., Fullaondo,A., Galan,J., Diaz-Uriarte,R., Malumbres,M., Field,S.J. and Zubiaga,A.M. (2008) E2F2 represses cell cycle regulators to maintain quiescence. Cell Cycle, 7, 3915-3927.

37. Aerts,S., Van Loo,P., Thijs,G., Mayer,H., de Martin,R., Moreau,Y. and De Moor,B. (2005) TOUCAN 2: the all-inclusive open source workbench for regulatory sequence analysis. Nucleic Acids Res., 33, W393-W396.

38. Laresgoiti,U., Apraiz,A., Olea,M., Mitxelena,J., Osinalde,N., Rodriguez,J.A., Fullaondo,A. and Zubiaga,A.M. (2013) E2F2 and CREB cooperatively regulate transcriptional activity of cell cycle genes. Nucleic Acids Res., 41, 10185-10198.

39. Thangavel,C., Boopathi,E., Ertel,A., Lim,M., Addya,S., Fortina,P., Witkiewicz,A.K. and Knudsen,E.S. (2013) Regulation of miR106b cluster through the RB pathway: mechanism and functional targets. Cell Cycle, 12, 98-111.

40. Wells,J., Boyd,K.E., Fry,C.J., Bartley,S.M. and Farnham,P.J. (2000) Target gene specificity of E2F and pocket protein family members in living cells. Mol. Cell. Biol., 20, 5797-5807.

41. Heo,I., Joo,C., Cho,J., Ha,M., Han,J. and Kim,V.N. (2008) Lin28 mediates the terminal uridylation of let-7 precursor MicroRNA. Mol. Cell, 32, 276-284.

42. Newman,M.A., Thomson,J.M. and Hammond,S.M. (2008) Lin-28 interaction with the Let-7 precursor loop mediates regulated microRNA processing. RNA, 14, 1539-1549.

43. Piskounova,E., Viswanathan,S.R., Janas,M., LaPierre,R.J., Daley,G.Q., Sliz,P. and Gregory,R.I. (2008) Determinants of microRNA processing inhibition by the developmentally regulated RNA-binding protein Lin28. J. Biol. Chem., 283, 21310-21314.

44. Rybak,A., Fuchs,H., Smirnova,L., Brandt,C., Pohl,E.E., Nitsch,R. and Wulczyn,F.G. (2008) A feedback loop comprising lin-28 and let-7 controls pre-let-7 maturation during neural stem-cell commitment. Nat. Cell Biol., 10, 987-993.

45. Viswanathan,S.R., Daley,G.Q. and Gregory,R.I. (2008) Selective blockade of microRNA processing by Lin28. Science, 320, 97-100.

46. Chang,T.C., Zeitels,L.R., Hwang,H.W., Chivukula,R.R., Wentzel,E.A., Dews,M., Jung,J., Gao,P., Dang,C.V., Beer,M.A. et al. (2009) Lin-28B transactivation is necessary for Myc-mediated let-7 repression and proliferation. Proc. Natl. Acad. Sci. U.S.A., 106, 3384-3389.

47. Bretones,G., Delgado,M.D. and Leon,J. (2015) Myc and cell cycle control. Biochim. Biophys. Acta, 1849, 506-516.

48. DeGregori,J. and Johnson,D.G. (2006) Distinct and overlapping roles for E2F family members in transcription, proliferation and apoptosis Curr. Mol. Med., 6, 739-748.

49. Martelli,F. and Livingston,D.M. (1999) Regulation of endogenous E2F1 stability by the retinoblastoma family proteins. Proc. Natl. Acad. Sci. U.S. A., 96, 2858-2863.

50. Sandoval,J., Rodríguez,J.L., Tur,G., Serviddio,G., Pereda,J., Boukaba,A., Sastre,J., Torres,L., Franco,L. and López-Rodas,G.
(2004) RNAPol-ChIP: a novel application of chromatin immunoprecipitation to the analysis of real-time gene transcription. Nucleic Acids Res., 32, e88.

51. Aksoy,O., Chicas,A., Zeng,T., Zhao,Z., McCurrach,M., Wang,X. and Lowe,S.W. (2012) The atypical E2F family member E2F7 couples the p53 and RB pathways during cellular senescence. Genes Dev., 26, 1546-1557.

52. Johnson,C.D., Esquela-Kerscher,A., Stefani,G., Byrom,M., Kelnar,K., Ovcharenko,D., Wilson,M., Wang,X., Shelton,J., Shingara,J. et al. (2007) The let-7 microRNA represses cell proliferation pathways in human cells. Cancer Res., 67, 7713-7722.

53. Kim,H., Huang,W., Jiang,X., Pennicooke,B., Park,P.J. and Johnson,M.D. (2010) Integrative genome analysis reveals an oncomir/oncogene cluster regulating glioblastoma survivorship. Proc. Natl. Acad. Sci. U.S.A., 107, 2183-2188.

54. Kota,J., Chivukula,R.R., O’Donnell,K.A., Wentzel,E.A., Montgomery,C.L., Hwang,H.W., Chang,T.C., Vivekanandan,P., Torbenson,M., Clark,K.R. et al. (2009) Therapeutic microRNA delivery suppresses tumorigenesis in a murine liver cancer model. Cell, 137, 1005-1017.

55. Lee,J.J., Drakaki,A., Iliopoulos,D. and Struhl,K. (2012) MiR-27b targets PPARgamma to inhibit growth, tumor progression and the inflammatory response in neuroblastoma cells. Oncogene, $\mathbf{3 1}$ $3818-3825$.

56. Schultz,J., Lorenz,P., Gross,G., Ibrahim,S. and Kunz,M. (2008) MicroRNA let-7b targets important cell cycle molecules in malignant melanoma cells and interferes with anchorage-independent growth. Cell Res., 18, 549-557.

57. Suh,S.S., Yoo,J.Y., Nuovo,G.J., Jeon,Y.J., Kim,S., Lee,T.J., Kim,T., Bakacs,A., Alder,H., Kaur,B. et al. (2012) MicroRNAs/TP53 feedback circuitry in glioblastoma multiforme. Proc. Natl. Acad. Sci. U.S.A., 109, 5316-5321.

58. Wang,Y., Rathinam,R., Walch,A. and Alahari,S.K. (2009) ST14 (suppression of tumorigenicity 14) gene is a target for miR-27b, and the inhibitory effect of ST14 on cell growth is independent of miR-27b regulation. J. Biol. Chem., 284, 23094-23106.

59. He,L., Thomson,J.M., Hemann,M.T., Hernando-Monge,E., Mu,D., Goodson,S., Powers,S., Cordon-Cardo,C., Lowe,S.W., Hannon,G.J. et al. (2005) A microRNA polycistron as a potential human oncogene. Nature, 435, 828-833.

60. Mitxelena,J., Osinalde,N., Arizmendi,J.M., Fullaondo,A. and Zubiaga,A.M. (2012) Proteomic approaches to unraveling the $\mathrm{RB} / \mathrm{E} 2 \mathrm{~F}$ regulatory pathway. In: Man, TK and Flores, RJ (eds). Proteomics-Human Diseases and Protein Functions, INTECH Open Access Publisher, Rijeka, 135-160.

61. Salvatori,B., Iosue,I., Mangiavacchi,A., Loddo,G., Padula,F., Chiaretti,S., Peragine,N., Bozzoni,I., Fazi,F. and Fatica,A. (2012) The microRNA-26a target E2F7 sustains cell proliferation and inhibits monocytic differentiation of acute myeloid leukemia cells. Cell Death Dis., 3, e413. 